\section{SARCOMA DE EWING CONGÊNITO: RELATO DE UM CASO}

\section{CONGENITAL EWING'S SARCOMA: A CASE REPORT}

\author{
Ana Maria Benvegnú ${ }^{1}$, Ana Paula Naspolini ${ }^{1}$, \\ Karen Regina Rosso Schons ${ }^{1}$, Cristiane Faccin Knob Horbach ${ }^{1}$, \\ André Avelino Costa Beber ${ }^{1}$
}

\begin{abstract}
RESUMO
O sarcoma congênito da família Ewing é uma doença extremamente rara, que se manifesta através do surgimento de uma massa e sinais sistêmicos como dor e febre. Cerca de 70 a $80 \%$ dos casos ocorrem antes dos 20 anos de idade. Histologicamente se caracteriza pela presença de pequenas células arredondadas e azuis. Além disso, pode se manifestar em tecidos moles ou osso e em qualquer parte do corpo, sendo a forma congênita mais comumente encontrada nas extremidades. O diagnóstico é estabelecido através de exames de imagem, histopatologia e imuno-histoquímica. Esses tumores costumam ser agressivos e apresentar elevada mortalidade. Aqui, relatamos o caso de um recém-nascido que apresentou uma massa no membro superior esquerdo diagnosticada como sarcoma congênito da família Ewing por biópsia cutânea por punch e pela presença do marcador CD99.
\end{abstract}

Palavras-chave: Diagnóstico; recém-nascido; Sarcoma de Ewing; prognóstico

\begin{abstract}
Congenital Ewing's sarcoma is an extremely rare disease, manifested by the appearance of a mass and systemic signs such as pain and fever. About $70-80 \%$ of cases occur before 20 years of age. This condition is histologically characterized by the presence of small round blue cells and can manifest in bone or soft tissue, anywhere in the body, with the congenital form being the most commonly found in the extremities. The diagnosis is established by imaging, histopathology, and immunohistochemistry. These tumors tend to be aggressive and have high mortality rates. Here, we report the case of a newborn who presented a mass in the left upper limb diagnosed as congenital Ewing's sarcoma by punch skin biopsy and the presence of the CD99 marker.
\end{abstract}

Keywords: Diagnosis; newborn; Ewing's sarcoma; prognosis

Os tumores da família Ewing podem ocorrer em qualquer idade; porém, cerca de 70 a $80 \%$ dos casos ocorrem até os 20 anos e $14 \%$ deles são diagnosticados em idade inferior a 5 anos; além disso, a forma congênita é extremamente rara $^{1-3}$. São histologicamente caracterizados pela presença de pequenas células arredondadas e azuis em tecidos moles e ossos e incluem sarcoma de Ewing, tumor neuroectodérmico primitivo periférico, neuroepitelioma periférico e tumor de Askin ${ }^{1,2}$. O sarcoma de Ewing é pouco diferenciado ou indiferenciado, apresentando prognóstico reservado. Apresentamos um caso de sarcoma de Ewing congênito diagnosticado através de biópsia cutânea por punch.

\section{RELATO DO CASO}

Paciente recém-nascido do sexo masculino, sem acompanhamento pré-natal. Nasceu pós-termo, por parto cesáreo em apneia. Ao nascimento apresentou massa hipervascularizada e hiperemiada em membro superior esquerdo (MSE), estendendo-se para a região axilar, cervical e torácica
Clin Biomed Res. 2017;37(3):263-265

1 Ambulatório de Dermatologia, Hospital Universitário de Santa Maria, Universidade Federal de Santa Maria (UFSM). Santa Maria, RS, Brasil.

Autor correspondente: Ana Maria Benvegnú anabenvegnu@yahoo.com.br Ambulatório de Dermatologia, Hospital Universitário de Santa Maria, Universidade Federal de Santa Maria Av. Roraima, 1000, Prédio 22, Camobi. 97105-340, Santa Maria, RS, Brasil. 
(Figura 1). A mãe era hígida e negava exposição à irradiação e a produtos químicos na gestação. Foi realizada tomografia computadorizada (TC), a qual revelou massa de $10 \times 9 \mathrm{~cm}$ em MSE com acometimento da porção proximal do úmero, estendendo-se para a área axilar e torácica esquerdas, sem envolvimento de gradil costal. Desde o pós-parto imediato estava em uso de ampicilina e gentamicina devido a infecção neonatal. Na sequência, realizou ecocardiograma que evidenciou fluxo anterógrado turbulento em tronco pulmonar e ramo pulmonar direito. Nesse ecocardiograma, não foi possível visualizar o ramo pulmonar esquerdo, sendo sugerida como causa compressão tumoral. A TC de abdome demonstrou hepatoesplenomegalia, sem outras alterações. Optou-se pela realização de biópsia de

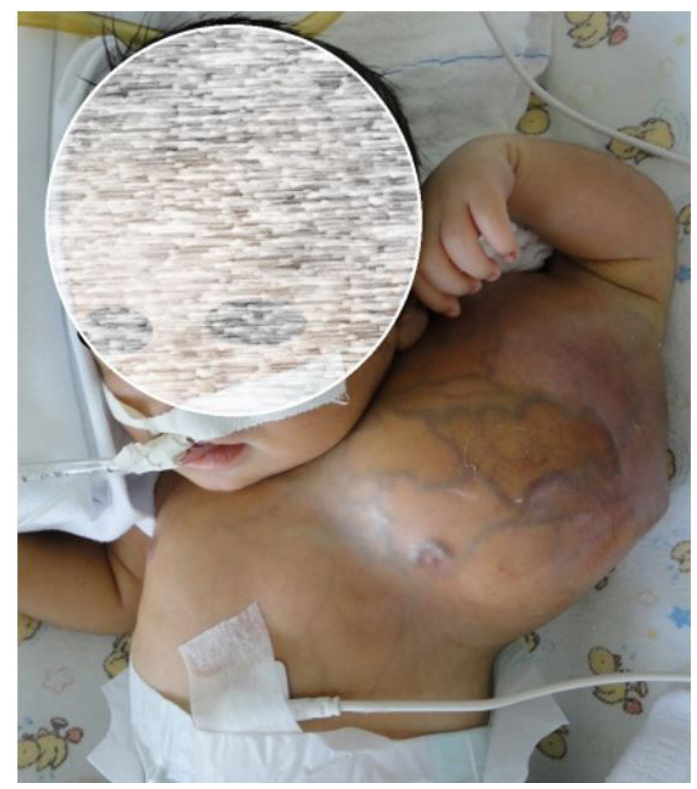

Figura 1: Massa hiperemiada e hipervascularizada em membro superior esquerdo, estendendo-se para a região axilar, cervical e torácica. pele por punch $\mathrm{n}^{\circ} 5 \mathrm{~mm}$ guiada por ultrassonografia, a qual revelou neoplasia de pequenas células redondas e azuis, apoptose moderada e áreas de necrose. A imuno-histoquímica afastou rabdomiossarcoma ou linfoma linfoblástico e evidenciou positividade para marcadores CD99 (com clone 013), positividade difusa para vimentina e focal para citoqueratinas (Figura 2). Os achados corroboraram o diagnóstico de tumor da família Ewing. O paciente realizou um ciclo de quimioterapia com carboplatina e etoposide, evoluindo com pancitopenia severa. Apesar das múltiplas drogas em uso (vancomicina, meropenem e anfotericina B) e das múltiplas tentativas de recuperação através de infusão de plaquetas, glóbulos e plasma, o paciente faleceu com 1 mês de vida devido à neutropenia e à infecção neonatal tardia.

\section{DISCUSSÃO}

Os tumores congênitos da família Ewing são caracterizados histologicamente por pequenas células azuis e arredondadas, podendo se desenvolver em tecidos moles ou ossos. A forma congênita pode se manifestar em qualquer parte do corpo, porém observamos maior incidência no tronco ${ }^{1}$. Esses tumores acometem mais frequentemente pacientes na segunda década de vida, cerca de $80 \%$ são encontrados abaixo dos 20 anos, sendo que a forma congênita é extremamente rara, visto que até o momento foram encontrados relatos de 17 casos na literatura ${ }^{1-3}$. Eles se originam do neuroectoderma e são compostos por células neuroepiteliais indiferenciadas ou pouco diferenciadas com capacidade de diferenciação em células neuronais, neurogliais ou outras formas mesenquimais ${ }^{2}$. Apresentam-se como uma massa e podem estar associados a sintomas sistêmicos como dor. O seu diagnóstico é baseado em exames de imagem e pelas características da histopatologia e da imuno-histoquímica. Durante o pré-natal já é possível realizar o diagnóstico através dos exames ultrassonográficos, o que pode ser útil no preparo da gestante ou para uma possível intervenção intrauterina.

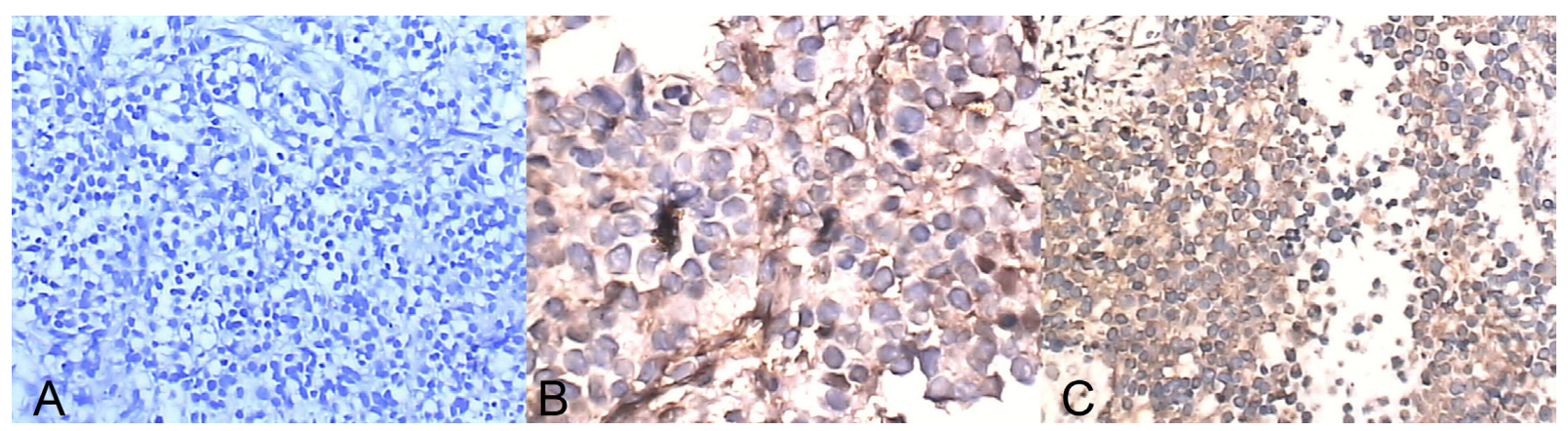

Figura 2: (A) Pequenas células redondas e azuis (HE 100x); (B) e (C) Positividade para CD99 (IHQ 400x e 100x). 
Os exames de imagem não mostram características específicas desse tumor, fazendo-se necessária a realização de histopatologia e imuno-histoquímica. A histologia se caracteriza por pequenas células azuis e arredondadas. Cerca de $90 \%$ dos casos apresentam translocação cromossômica, como t $(11 ; 22)$ (q24;q12), t(21;22)(q22; 12$)$ ou $\mathrm{t}(7 ; 22)(\mathrm{p} 22 ; \mathrm{q} 12)$, com envolvimento da fusão dos genes EWS/FLI-1. No nosso caso, a translocação cromossômica não foi avaliada, devido ao fato de esse exame não estar disponível no hospital em estudo. A presença do marcador imuno-histoquímico CD99 associado à translocação cromossômica possui alta sensibilidade e especificidade para o diagnóstico ${ }^{2,4-8}$. Com a elevada especificidade da translocação e os poucos fatores de risco encontrados, sugere-se uma predisposição genética como a origem do tumor ${ }^{8}$. Os tumores congênitos possuem comportamento agressivo, com rápida progressão, alta recorrência e risco de metástases. Esse prognóstico torna-se ainda mais sombrio quando, ao diagnóstico, o paciente apresenta-se com metástases ${ }^{1,7}$. $O$ tratamento consiste na ressecção cirúrgica radical seguida de combinações de quimioterapia com ou sem radioterapia. Ainda é possível o transplante de células-tronco associado à quimioterapia precoce ${ }^{7}$.
Os esquemas quimioterápicos mais utilizados são com vincristina, ciclofosfamida e doxorrubicina, alternados com etoposide e ifosfamida ${ }^{2,4-7}$.

Foram encontrados 17 relatos de casos de tumores congênitos da família Ewing. Em seu artigo, Shu-Guang Jin et al. ${ }^{2}$ apresentam uma tabela com os casos relatados até o ano de 2013 , totalizando 14 casos, incluindo o desses autores. Além desse trabalho, ainda foram encontrados o caso de tumor congênito da família Ewing paraespinhal ${ }^{7}$, o caso de tumor congênito da família Ewing na ulna ${ }^{6}$ e o caso de tumor congênito da família Ewing na escápula ${ }^{9}$. Dos casos relatados, foi constatado prognóstico reservado, com elevada mortalidade e recidiva. Cerca de $38 \%$ dos pacientes apresentaram sobrevida superior a 1 ano de idade. Porém, dos pacientes que completaram 1 ano de idade, $57 \%$ foram a óbito posteriormente ${ }^{2,6,7,9}$.

Apresentamos um caso raro de tumor congênito da família Ewing diagnosticado precocemente por biópsia cutânea por punch, a qual demonstrou alterações histológicas características, e pela presença do marcador imuno-histoquímico CD99. $\mathrm{O}$ paciente recebeu tratamento quimioterápico de imediato, porém sem resposta, demonstrando a gravidade da doença.

\section{REFERÊNCIAS}

1. Saito $Y$, Matsuzaki $A$, Suminoe A, Koga $Y$, Kurata H, Oda Y, et al. Congenital Ewing sarcoma in retroperitoneum with multiple metastases. Pediatr Blood Cancer 2008;51(5):698-701. PMid:18623202. http://dx.doi.org/10.1002/pbc.21678.

2. Jin SG, Jiang XP, Zhong L. Congenital Ewing's Sarcoma/peripheral primitive neuroectodermal tumor: a case report and review of the literature. Pediatr Neonatol. 2016;57(5):4369. PMid:24480101. http://dx.doi. org/10.1016/j.pedneo.2013.11.002

3. Goyal S, Biswas A, Gupta R, Mohanti BK. Congenital peripheral primitive neuroectodermal tumor: a case treated successfully with multimodality treatment. J Egypt Natl Canc Inst. 2014;26(4):219-24. PMid:25440226. http://dx.doi.org/10.1016/j. jnci.2014.09.002.

4. Rosa M, Mohammadi A, Campos M, García-García I, Correa-Rivas MS. Congenital EWS/pPNET presenting as a neck mass. Pediatr Blood Cancer. 2009;53(4):678-9. PMid:19492319. http://dx.doi.org/10.1002/pbc.22073.

5. Kim SY, Tsokos M, Helman LJ. Dilemmas associated with congenital ewing sarcoma family tumors. $J$ Pediatr Hematol Oncol. 2008;30(1):47. PMid:18176172. http://dx.doi. org/10.1097/MPH.0b013e31815cf71f.

6. Okpokowuruk FS, Oloyede I. Congenital Ewing's Sarcoma in a neonate in Uyo - a case report. Pan Afr Med J. 2013;15:90. PMid:24198886. http://dx.doi. org/10.11604/pamj.2013.15.90.2219.
7. Ban SP, Park SH, Wang KC, Cho BK, Phi JH, Lee JY, et al. Congenital paraspinal Ewing sarcoma family of tumors with an epidural extension. J Clin Neurosci. 2010;17(12):1599601. PMid:20817468. http://dx.doi. org/10.1016/j.jocn.2010.03.059.

8. Cope JU, Reaman GH, Tonning JM. Medication exposures and subsequent development of Ewing Sarcoma: a review of FDA adverse event reports. Sarcoma. 2015;2015(2015):948159.

9. Jinkala SR, Basu D, Mathath $D$, Dubashi B, Bhaumik A. A rare case of congenital Ewing sarcoma/PNET of the scapula. J Pediatr Hematol Oncol. 2014;36(2):e134-5. PMid:24072238. http://dx.doi.org/10.1097/ MPH.0000000000000004. 